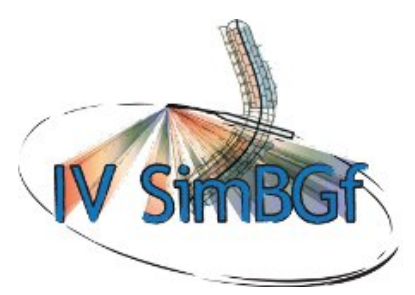

\title{
FLUXO DE TRABALHO NA AVALIAÇÃO PETROFÍSICA DE POÇOS
}

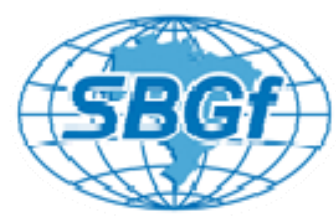

\author{
1. Ronaldo Cavalcante Freire / PETROBRAS/E\&P-EXP \\ 2. Antônio Carlos de Freitas Nascimento / PETROBRAS/E\&P-EXP \\ 3. Paulo Sérgio Denicol / PETROBRAS/E\&P-EXP \\ 4. Carlos Francisco Beneduzi / PETROBRAS/E\&P-EXP
}

Copyright 2010, SBGf - Sociedade Brasileira de Geofísica

Este texto foi preparado para a apresentação no IV Simpósio Brasileiro de Geofísica, Brasília, 14 a 17 de novembro de 2010. Seu conteúdo foi revisado pelo Comitê Técnico do IV SimBGf, mas não necessariamente representa a opinião da SBGf ou de seus associados. É proibida a reprodução total ou parcial deste material para propósitos comerciais sem prévia autorização da SBGf.

\section{Resumo}

Esse trabalho descreve um fluxo de trabalho de avaliação petrofísica aplicado a poços de prospecção de hidrocarbonetos através da utilização de dados de perfis elétricos. Além dos dados de perfis, crossplots, histogramas, modelo mineralógico probabilístico etc. auxiliam o fluxo de trabalho dando suporte para uma avaliação petrofísica integrada.

\section{Introdução}

A petrofísica estuda as propriedades físicas e químicas das rochas e fluidos. Na indústria do petróleo e gás, a avaliação petrofísica envolve a definição e distribuição dos fluidos (óleo, gás ou água) e suas interações com o sistema poroso presente na rocha, com o objetivo de identificar a presença de hidrocarbonetos em reservatórios porosos e permeáveis incorporando volumes recuperáveis de petróleo nas reservas provadas.

Essa avaliação consiste em integrar dados de poços, adquiridos a partir de ferramentas adequadas, e auxiliar 0 entendimento do comportamento de reservatórios, o processo de decisão sobre o aproveitamento e abandono de poços e áreas exploratórias e o gerenciamento de campos em produção, baseado nas características petrofísicas. A interação entre as equipes multidisciplinares como geólogos/geofísicos e engenheiros tem papel fundamental nesses processos e nas estimativas de capacidade de armazenamento de fluidos e na definição dos melhores métodos de escoamento e de recuperação de óleo e gás.

Este artigo tem como objetivo apresentar o fluxo de trabalho aplicado em poços através de técnicas e métodos, com a finalidade de se obter dados e parâmetros petrofísicos confiáveis a serem utilizados como input na avaliação petrofísica integrada.

\begin{abstract}
Metodologia
O fluxo de trabalho (workflow) (Figura 1) utilizado em uma avaliação petrofísica é baseado nas respostas dos perfis elétricos (gamma ray, ressonância magnética, resistividade, densidade, neutrão, sônico, caliper, fator fotoelétrico etc.) e utiliza informações de diversos gráficos (crossplots) e histogramas, podendo fornecer dados sobre a composição da matriz dos reservatórios, a qualidade de reservatórios/perfis, estimativa da porosidade e dos parâmetros elétricos e saturações de fluidos.
\end{abstract}

Os crossplots e histogramas usualmente utilizados em uma avaliação petrofísica e que podem fornecer importantes subsídios para o fluxo de trabalho são mostrados na Figura 1 e no tópico de resultados.

Através dos perfis dos poços também é possível modelar ou simular um modelo mineralógico probabilístico que estima os volumes dos minerais presentes no poço. As estimativas desses minerais podem auxiliar na identificação do tipo do reservatório, das fácies reservatório e não-reservatório e dos processos diagenéticos que podem ter afetado a qualidade do reservatório como possíveis barreiras de permeabilidades (cimentação e presença de argilas).

\section{Resultados}

Um exemplo dos perfis elétricos adquiridos em poços é apresentado na Figura 2. No track 1 estão as curvas de Gamma Ray (GR) e capiler (HCAL), no track 2 a profundidade do poço, no track 3 as curvas de resistividades rasa (RXOZ) e profunda (RLA5) e no track 4 as curvas de densidade (RHOZ), fator fotoelétrico (PEFZ), neutrão (NPOR) e sônico (DT4P).

Crossplots e histogramas aliados as respostas dos perfis elétricos podem ser utilizados como diagnósticos ou indicadores da presença de reservatórios, da qualidade desses reservatório, do tipo litológico e estimativa de porosidade/permeabilidade, da identificação da presença e saturações dos fluidos presentes na rocha. 
- Identificação litológica, porosidade/permeabilidade e identificação de gás

Para a identificação da composição da matriz do reservatório podem ser utilizados os gráficos Densidade (DEN) x Neutrão (NEU), Densidade de Matriz Aparente (Rhoma) x Seçao Cruzada Volumétrica Aparente (Uma).

O gráfico DEN x NEU (Figura 3) auxilia na identificação, de acordo com as linhas de matriz e a distribuição dos pontos no gráfico, do tipo de matriz da rocha (calcítica, dolomítica e arenítica). A indicação de presença de gás no reservatório também pode ser vista nesse crossplot quando os pontos se distribuem acima da linha do arenito (Figura 3).

O gráfico Rhoma $x$ Uma auxilia as informações de tipo de matriz sugerida pelo gráfico DEN $x$ NEU e fornece informação qualitativa sobre as condições permo-porosas do reservatório. Quando observa-se altos valores de Uma pode ser sugerido uma invasão de baritina do fluido de perfuração na formação, indicando que o reservatório possui qualitativamente uma boa condição de permoporosidade (Figura 4).

\section{- Identificação de reservatórios}

A delimitação das zonas reservatório e não reservatório é feita analisando-se conjuntamente as respostas dos perfis elétricos. O perfil de GR é analisado inicialmente, e depois observado o comportamento das curvas de resistividade, assim como outras curvas (DEN, NEU, sônico, ressonância magnética) para observar a influência sobre as porosidades, água retida nos poros (nas argilas ou nos microporos) para identificação dos intervalos reservatórios (Figura 5).

\section{- Identificação qualitativa de hidrocarbonetos}

A partir do Hingle Plot (crossplot de DEN x 1/Rt^0.5) podem ser feitas estimativas das saturações de água dos reservatórios em análise, indicando qualitativamente intervalos portadores de hidrocarbonetos e/ou de água conforme apresentado na Figura 6 (Rt - resistividade da formação).

O expoente de cimentação $(\mathrm{m})$ também pode ser utilizado como um indicador qualitativo da presença de hidrocarboneto. O M plot (Figura 7) trata-se de um gráfico onde são mostradas as variações do expoente de cimentação, da fórmula de Archie (Archie, 1942), com a variação da porosidade.

Em arenitos com porosidades constantes portadores de água o $m$ tende a permanecer constante, enquanto que na presença de hidrocarbonetos a variação de $m$ com a porosidade define uma reta cuja inclinação é mais elevada, devido a restrição à corrente elétrica e o aumento da tortuosidade.

\section{- Cálculo de Saturação de Água}

Diversas equações podem ser utilizadas para o cálculo de saturações de água nos reservatórios. A principal delas é a equação (1) de Archie (Archie, 1942) mostrada abaixo.

$$
S w=\sqrt[n]{\frac{R w \cdot a}{R t \cdot \phi^{m}}}
$$

onde: $\mathrm{Sw}=$ saturação de água; $\mathrm{m}$ = expoente de cimentação; $\mathrm{n}=$ expoente de saturação; $\mathrm{Rw}=$ resistividade da água; $\mathrm{Rt}=$ resistividade da formação; $\mathrm{a}=$ 1 (coeficiente de tortuosidade).

As outras equações de Sw propostas por outros autores foram sugeridas com o intuito de suprir deficiências da equação de Archie em algumas situações, como, a presença de argilosidade, de fraturas, de porosidade vugular etc.

Fatores importantes para o cálculo de Sw que devem ser levados em conta e tratados cuidadosamente são: o cálculo da porosidade efetiva (Phie), da resistividade da água da formação (Rw) e dos parâmetros elétricos (m e n).

A partir da distribuição de T2 (tempo de relaxamento) do perfil de ressonância magnética também é possível a estimativa da saturação de água irredutível presente nos reservatórios conforme apresentado na Figura 8.

Através dos perfis de poços também é possível modelar ou simular um modelo mineralógico probabilístico que estima os volumes dos minerais presente no poço. A estimativa desses minerais auxilia na identificação do tipo do reservatório, das fácies reservatório e não-reservatório e dos processos diagenéticos que podem ter afetado a qualidade do reservatório como possíveis barreiras de permeabilidades (cimentação e presença de argilas) (Figura 9).

\section{Discussão e Conclusões}

Ao final de uma avaliação petrofísica totaliza-se os dados com o intuito de expor valores (porosidade, saturação de água e espessura porosa com óleo (net pay)) que expressam o potencial petrolífero do reservatório em estudo. Para totalizar os dados são definidos parâmetros de corte (cut-off's) dessas variáveis, buscando sua viabilidade econômica e operacional.

Os valores de saturação de água podem ser obtidos pela equação de Archie e suas derivações, pela distribuição de T2 do perfil de ressonância magnética e através da porosidade exportada do modelo mineralógico utilizando a equação de Sw escolhida.

De acordo com a definição dos parâmetros de corte (porosidade e saturação de água) pode ser calculado o net pay do reservatório que resulta em um HPhiSO, a partir do produto da porosidade, saturação de óleo e net pay, expressando também o potencial do reservatório. 


\section{Agradecimentos}

Aos autores do trabalho Antônio Carlos de Freitas Nascimento, Paulo Sérgio Denicol e Carlos Francisco Beneduzi (E\&P-EXP/AFOE/AFP) pela ajuda e orientação técnica. A PETROBRAS pelo apoio a realização desse trabalho.

\section{Referências}

Archie, G. E. 1942. The Electrical Resistivity Log as an Aid in Determining Some Reservoir Characteristics: Journal of Petroleum Technology, v. 5, p. 54-62.

Asquit, G. B. 1985. Handbook of Log Evaluation Techniques for Carbonate Reservoirs. Methods in Exploration Series No. 5. The American Association of Petroleum Geologists. Tulsa, Oklahoma. 47p.

Asquith, G. \& Krygowski, D. 2004. Basic Well Log Analysis: AAPG Methods in Exploration 16. The American Association of Petroleum Geologists. Oklahoma.

Coates, G. R., Xiao, L., Prammer, M. G. 1999. NMR Logging Principles \& Aplications. Halliburton Energy Services Publication H02308. 234 p.

Denicol, S. \& Nascimento, A. C. F. 2006. Avaliação de Reservatórios Complexos. VII Simpósio Técnico de Operações Geológicas, Macaé/RJ.

Hingle, A.T. 1959. The Use of Logs in Exploration Problems. SEG Paper. Los Angeles.

Nascimento, A. C. F., Borges, L. P., Denicol, P. S. 2006. Método para Determinação do Expoente de Saturação "n" com o Auxílio do Perfil de Ressonância Magnética. Nota Técnica PETROBRAS E\&P-EXP/AFOE/AFP.

Nurmi, R. D. 1984. Carbonate Pore Systems: Porosity/\{Permeability Relationships and Geological Analysis AAPG Annual Meeting, San Antonio (May 2023) in Asquit, G. B. 1985. Handbook of Log Evaluation Techniques for Carbonate Reservoirs. Methods in Exploration Series No. 5. The American Association of Petroleum Geologists. Tulsa, Oklahoma. 47p.

Rêgo, A. P. M., Neto, O. M., Peixoto, P. P. 2006 Utilizacao de Crossplots e Gradientes de Pressão na Avaliação e Interpretação Geológica da Área dos Poços 1-FAE-1D-AL e 1-FAE-2D-AL. Simpósio Técnico de Operações Geológicas - VII STOG. 


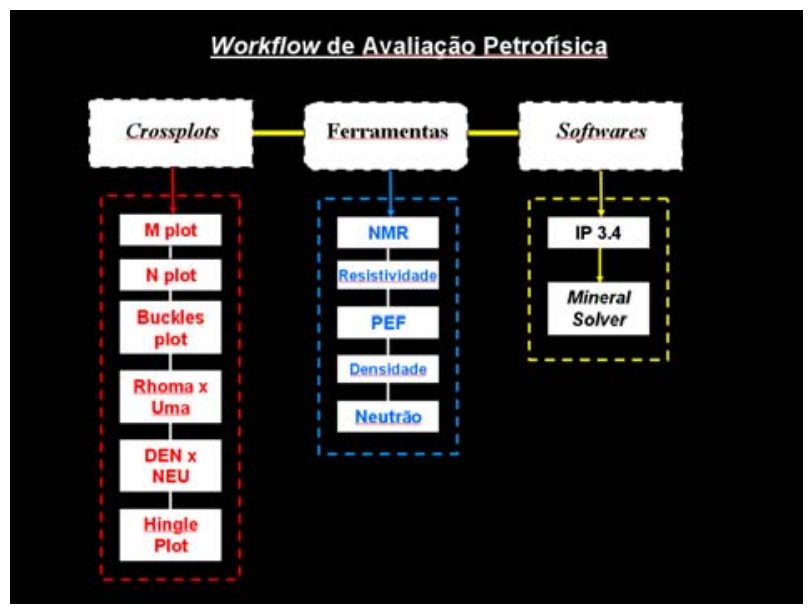

Figura 1 - Workflow de avaliação petrofísica.

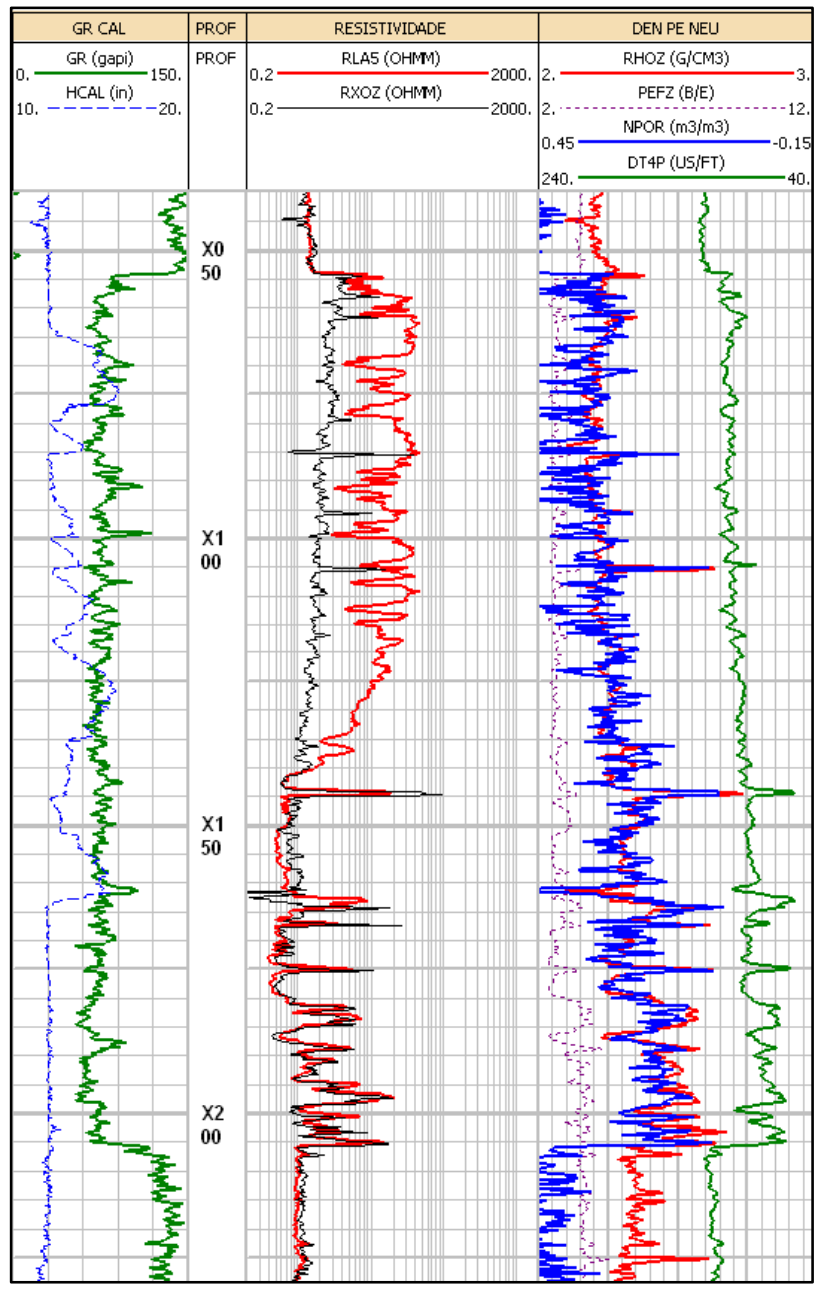

Figura 2 - Plot de um poço mostrando os perfis elétricos adquiridos. Track 1: GR e HCAL; Track 2: Profundidade; Track 3: RXOZ e RLA5; Track 4: RHOZ, PEFZ, NPOR e DT4P.

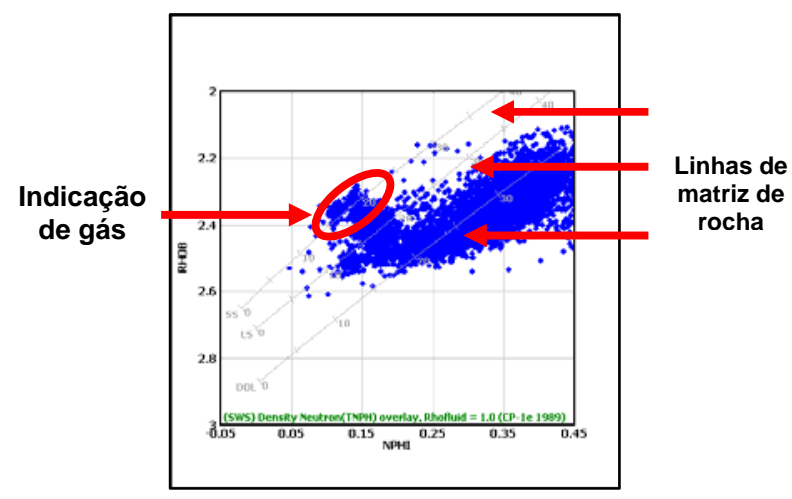

Figura 3 - Gráfico DEN x NEU com as linhas de matriz (superior: arenito; intermediária: calcita; inferior: dolomita).

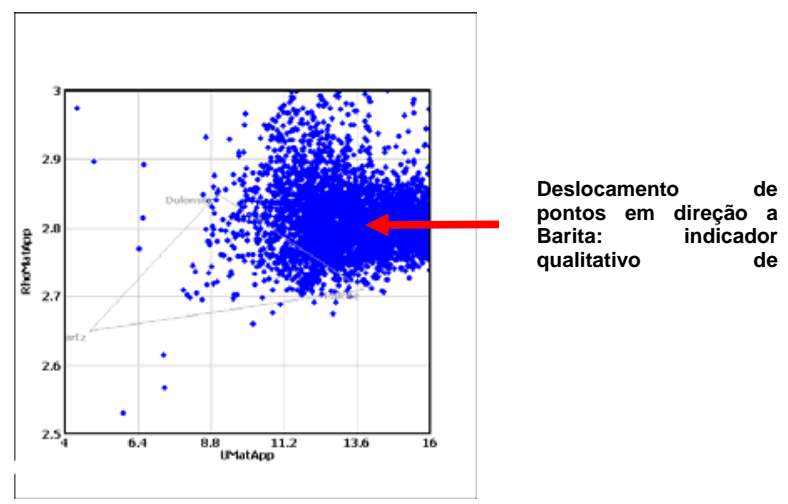

Figura 4 - Crossplot Rhoma x Uma identificando matriz calcítica com dolomitização e invasão de barita do fluido de perfuração. 


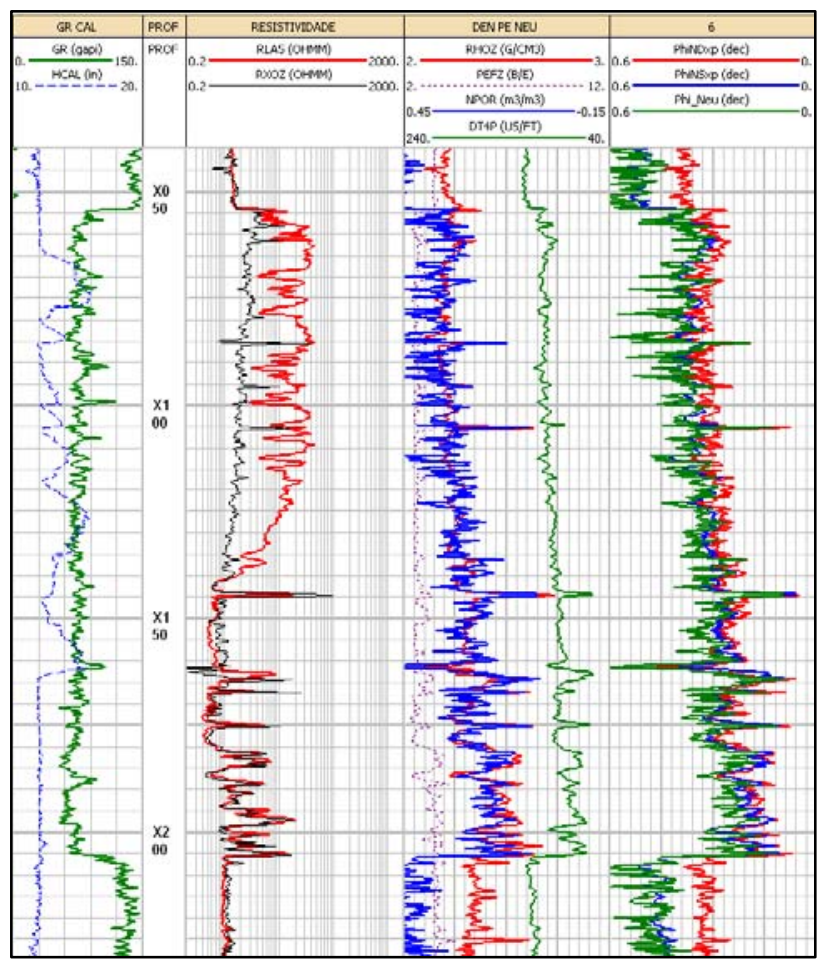

Figura 5 - Plot com as curvas de porosidade obtidas a partir das curvas de DEN, NEU e sônico que auxiliam na identifiação de reservatórios com hidrocarbonetos. Curvas: PhiNDxp, PhiNSxp e Phi_Neu (Track 4).

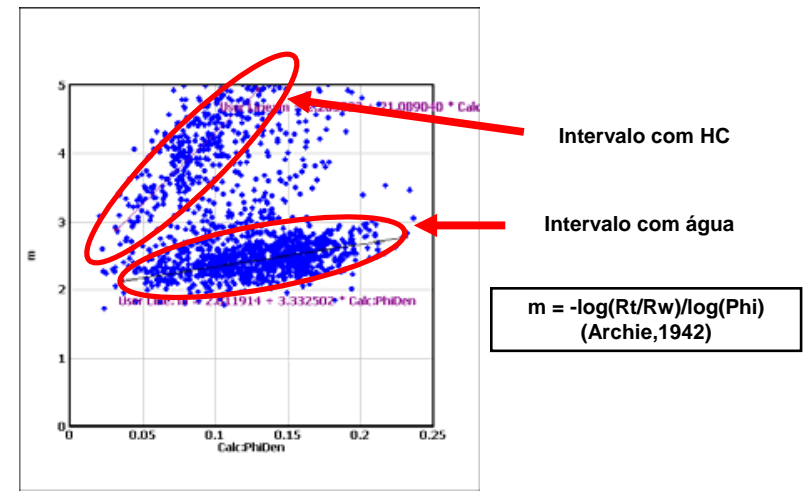

Figura 7 - M plot mostrando a distribuição dos pontos e as retas representativas das distintas fácies reservatório em estudo, indicando intervalo com hidrocarboneto e com água.

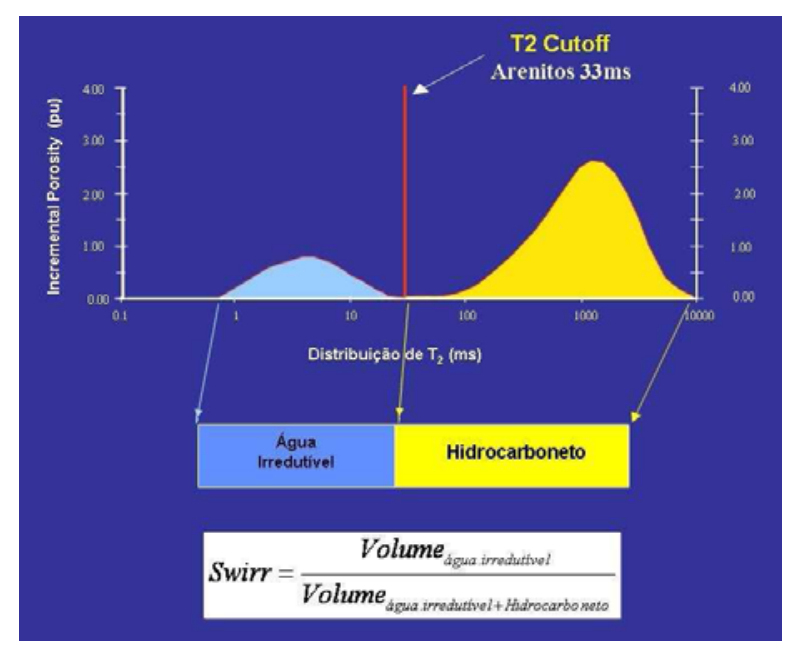

Figura 8 - Cálculo de saturação de água (Swirr) através da distribuição de T2 do perfil de ressonância magnética.

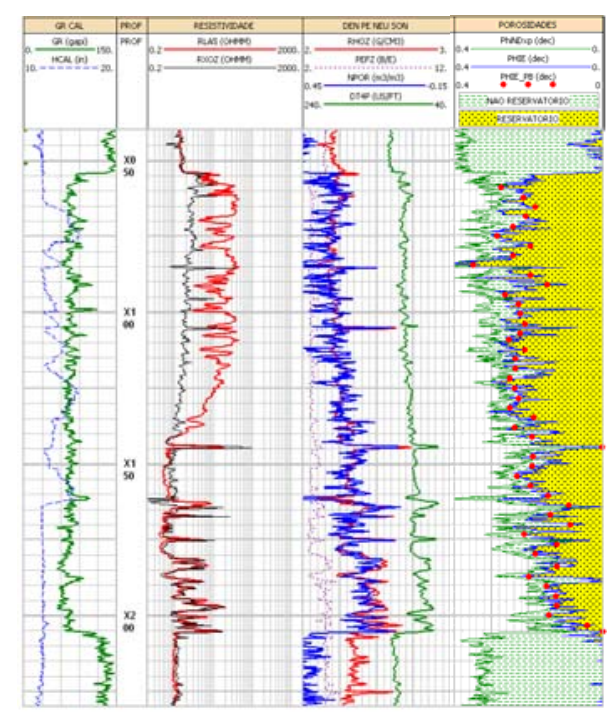

Figura 9 - Modelo mineralógico (Track 4) probabilístico com estimativa dos volumes dos minerais presentes. 
IV Simpósio Brasileiro da SBGf - Brasília 2010 